\title{
Breakdown of Scaling and Friction Weakening in Intermittent Granular Flow
}

\begin{abstract}
A. Baldassarri ${ }^{1}$, M. A. Annunziata ${ }^{1}$, A. Gnoli ${ }^{1}$, G. Pontuale ${ }^{1,2}$ \& A. Petri ${ }^{1 *}$
Many materials are produced, processed and stored as grains, while granularity of matter can be crucial in triggering potentially catastrophic geological events like landslides, avalanches and earthquakes. The response of grain assemblies to shear stress is therefore of utmost relevance to both human and natural environment. At low shear rate a granular system flows intermittently by distinct avalanches. In such state the avalanche velocity in time is expected to follow a symmetrical and universal average behavior, whose dependence on the slip size reduces to a scale factor. Analyzing data from long lasting experiments, we observe a breakdown of this scaling: While in short slips velocity shows indeed a self-similar and symmetric profile, it does not in long slips. The investigation of frictional response in these different regimes evidences that this breakdown can be traced back to the onset of a friction weakening, which is of dynamical origin and can amplify instabilities exactly in this critical state, the most frequent state for natural hazards.
\end{abstract}

The way a granular medium responses to an applied shear stress reveals many of the peculiarities of this poorly comprehended "state" of matter ${ }^{1}$. When a granular bed is sheared slowly enough by an elastic medium driven at constant velocity, nor the shear stress neither the shear rate can be directly controlled from outside. Rather, the system sets itself in a state at the edge between jamming and mobility ${ }^{2-9}$, exhibiting intermittent flow also called stick-slip. This is an instance, among many others, of phenomena displaying intermittent and erratic activity, in the form of bursts, or avalanches, characterized by wild fluctuations of physical quantities also called crackling nois $e^{10}$. Examples include earthquakes ${ }^{11}$, fractures ${ }^{12}$, structural phase transitions ${ }^{13}$, plastic deformation ${ }^{14}$, and other diverse phenomena sharing several common statistical features. In particular, these phenomena often display long range correlations and self-similar distributions, i.e. power laws, of physical quantities over a wide range of values. In equilibrium physical systems such properties are usually observed in the vicinity of a critical phase transition. Therefore also in non equilibrium systems they are usually ascribed to the vicinity of some critical point ${ }^{10,15}$, which in granular media is identified with the jamming transition ${ }^{16}$. Consistently, critical transitions bring about the existence of universality classes, where systems microscopically very different display similar and universal statistical properties in their critical, i.e. self similar, dynamics.

Many features of the crackling noise, i.e. intermittency, and broad time and energy scales, are produced in simple laboratory experiments, where small beads of glass are slowly sheared by an elastic medium ${ }^{3}$. Such experiments not only supply relevant information for a better comprehension of the irregular dynamics of granular matter, but can also help to discriminate between critical systems, yielding important elements for the general understanding of off-equilibrium dynamics.

In order to compare different systems exhibiting critical dynamics, several quantities can be analyzed. Recent literature witnesses a surge of interest for the average avalanche time profile, or shape ${ }^{17}$. Introduced in the context of Barkhausen noise in ferromagnetic materials ${ }^{18}$, the average avalanche shape can provide a much sharper tool to test theory against experiments than the simple comparison of critical exponents characterizing probability distributions ${ }^{19}$. As shown for simple stochastic processes, the geometrical and scaling properties of the average shape depend on the temporal correlations of the dynamics ${ }^{20-22}$. Average avalanche shapes have been investigated for a variety of materials, well beyond magnetic systems ${ }^{17,23}$, ranging from intermetallic compounds and crystals ${ }^{24,25}$ to glassy and amorphous systems ${ }^{19,26-28}$ and, very recently, in cortical bursts ${ }^{29,30}$, in transport processes in living

${ }^{1}$ CNR - Istituto dei Sistemi Complessi, Dipartimento di Fisica, Università di Roma Sapienza, P.le A. Moro 2, I-00185, Roma, Italy. ${ }^{2}$ Consiglio per la Ricerca in Agricoltura e l'Analisi dell'Economia Agraria (CREA) - Research Centre for Forestry and Woods, Via Santa Margherita 80, I-52100, Arezzo, Italy. *email: alberto.petri@isc.cnr.it 

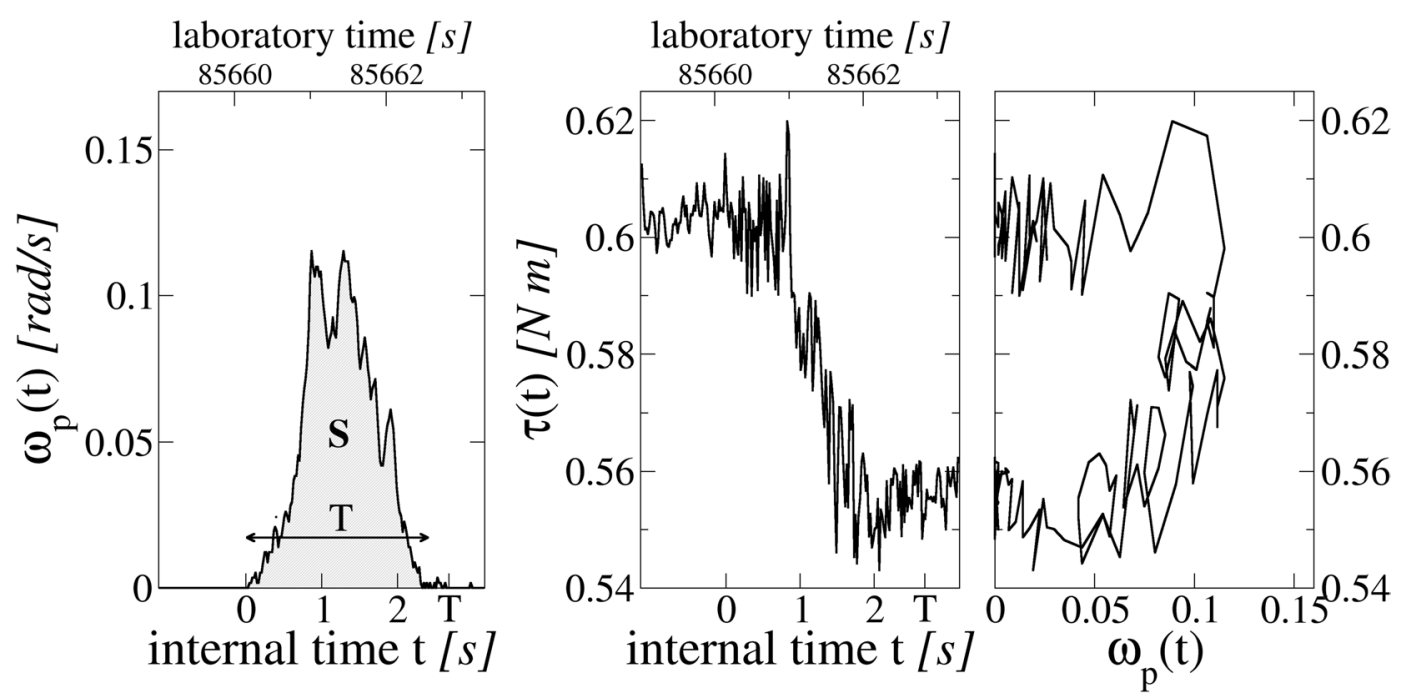

Figure 1. Sample of raw data for one of the six thousand slip events used in the present analysis. Left: instantaneous velocity of the slider versus time. The upper axis of the graph reports the total time elapsed from the beginning of the experiment, while the bottom axis indicates the internal avalanche time, starting from 0 when the slip begins, and ending at slip duration $T$. The area below the curve is the total slip size $S$. Center: Friction torque experienced by the slider in the same time window. Right: Behavior of friction torque vs instantaneous slider velocity for the same slip.

cells ${ }^{31}$, as well in ants ${ }^{32}$ and in human ${ }^{33}$ activity. Burst shape has also been investigated in stellar processes ${ }^{34}$, Earth's magnetospheric dynamics ${ }^{35}$, and earthquakes ${ }^{36}$.

We have acquired for the first time the slip velocity and the friction force in a sheared granular system, directly in the stick-slip phase, where it displays intermittent flow, and analyzed the corresponding average time profiles for different slip duration. In granular systems, the average time profile of velocity is expected to be symmetrical and invariant up to a rescaling ${ }^{37}$. On the contrary, in our study we observe the existence of a crossover from small slips, whose shapes satisfy the above properties, to large, non rescalable and non symmetrical slips. We identify this transition as a breakdown of the critical scaling, and show that the crossover corresponds to a characteristic speed marking a dynamical transition from weakening to thickening frictional behavior. These findings supply new essential elements to the formulation of novel and accurate dynamical models, with important impact on the understanding of related natural and technological issues. They also shed light on some apparently contradictory recent observations concerning avalanches of stress drop ${ }^{37}$ and energy drop rate ${ }^{38}$, conducted in continuous flow. While Denisov et al. ${ }^{37}$ observed avalanches displaying symmetrical and self-similar average shapes, Barés et al. ${ }^{38}$ found these properties only in sufficiently small avalanches. The investigation reported here, conducted in the intermittent state, shows that symmetric and asymmetric slips can both exist, depending on their duration and as a consequence of the rheological properties of the system.

\section{Results}

Scaling analysis. In our experiments (see Methods and Supplementry Information) an assembly of glass spheres laying in an annular channel is sheared by a horizontally rotating top plate. The plate is driven by a motor through a soft torsion spring. Although the angular speed of the motor is kept constant, the interaction between the plate and the granular medium is crucial in determining the instantaneous plate velocity. The intermittent dynamics, in which the plate performs highly irregular and intermittent motion, is approached when both driving speed and spring constant are low enough.

We have performed long lasting experimental runs in the intermittent, stick-slip regime, measuring the time behavior of the angular coordinate of the plate $\theta_{p}(t)$ and the plate angular velocity $\omega_{p}(t)$. We have then analyzed the stick-slip dynamics of the single slip event, illustrated in Fig. 1. The left panel reproduces the angular velocity during a slip. The motion can be described as a function of the internal avalanche time $t$, which starts at the beginning of the slip and ends when the system sticks. Each slip has its own duration $T$ and size $S$ (the grey area). The middle panel shows the corresponding frictional torque experienced by the plate in the same time interval. The right panel reports the instantaneous torque as function of the instantaneous plate velocity, showing how intricate and complex can be the relation relating these two quantities. Computing the average time profile of the velocity, or shape, requires to consider many slips of the same total duration $T$ and to perform averages as function of the internal avalanche time $t$, as described below. In a similar fashion one can consider the average friction time shape, i.e. the time behavior of the torque exerted in average by the granular medium on the plate at the internal time $t$ during a slip event of total duration $T$.

We have collected statistics from a large number of avalanches. The distribution of corresponding durations $T$ and sizes $S=\int_{0}^{T} \omega_{p}(t) d t$ are shown in Fig. 2. Both distributions exhibit a slow decay, close to a power law and 

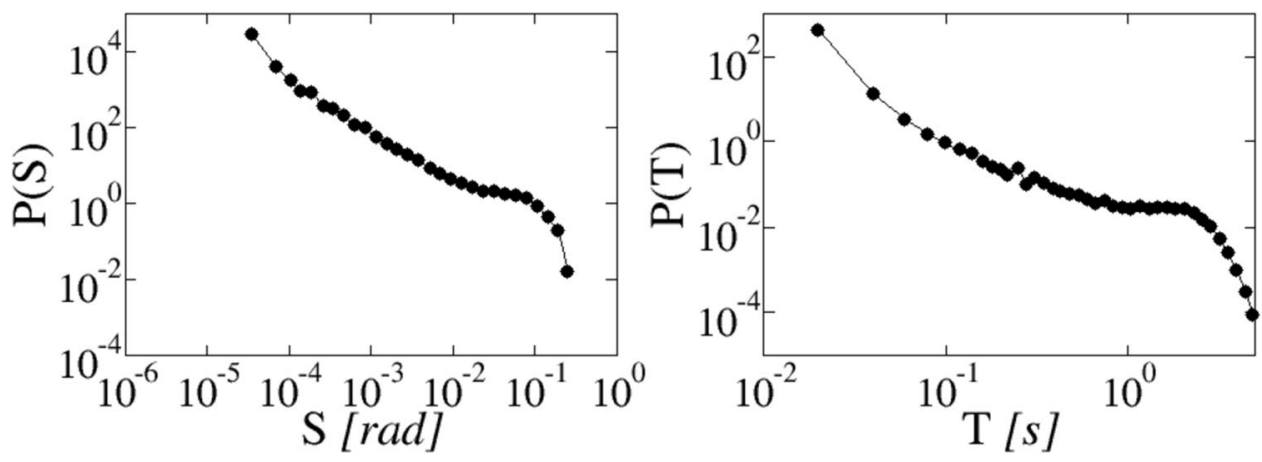

Figure 2. Probability distribution of slip extensions $S$ (left) and durations $T$ (right).

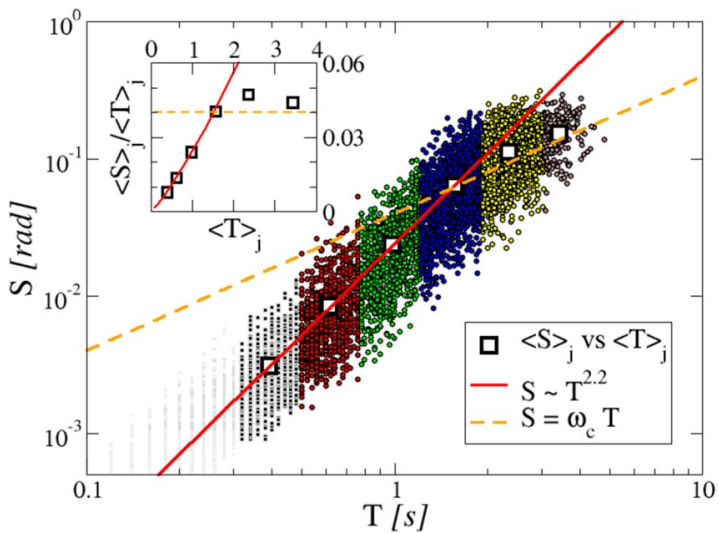

Figure 3. Scatter plot of size $S$ vs duration $T$ : each small point represents a single slip event. The events are divided into six classes of increasing duration, each one indicated with a different symbol and color (for the precise definition of the classes see SI). For each class $j=1, \ldots, 6$, the average duration $\langle T\rangle_{j}$ and an average slip $\langle S\rangle_{j}$ are computed, represented as white squares in the middle of each class. Inset: Average slip velocity $\langle S\rangle_{j} /\langle T\rangle_{j}$ for each class as a function of average duration $\langle T\rangle_{j}$ of the class. Lines (both in main plots and in inset), are guide to the eyes for: power law behavior (continuous line) $S \approx T^{2.2}$, and linear behavior (dashed line) $S=\omega_{c} T$ (where $\omega_{c}=0.04$, see text and Fig. 5 for definition).

terminating with a bulging cutoff at large values. Similar broad distributions are shared by other quantities ${ }^{4}$ like the plate velocity.

As recalled in the introduction, power law decays in distributions are generally considered the hallmark of critical phenomena. In this scenario, one expects to observe self-similar scaling relations in average quantities too. In particular, one can consider the average time profile of the velocity during an avalanche of duration, $T$ :

$$
\left\langle\omega_{p}(t)\right\rangle_{T}=\frac{1}{N_{T}} \sum_{i} \omega_{p}^{(i)}(t)
$$

where $\omega_{p}^{(i)}$ is the plate velocity during the $i_{t h}$ observed avalanche of duration $T$, whose total number is $N_{T}$, and $t$ is the internal time within the slip: $0<t<T$. Although the average velocity profile $\left\langle\omega_{p}(t)\right\rangle_{T}$ depends on both $t$ and $T$, criticality ${ }^{19,39,40}$ implies that an invariant function $\Omega$ exists, such that it can be expressed as:

$$
\left\langle\omega_{p}(t)\right\rangle_{T}=g(T) \Omega(t / T),
$$

where the function $g(T)$ determines how the average event size $\langle S\rangle_{T}$ scales with respect to the slip duration $T$. This scaling scenario is produced by several theoretical models of critical dynamics ${ }^{19}$. One paradigmatic model is the so called ABBM model ${ }^{41}$, proposed to describe the intermittent statistics of the electric noise induced during the hysteresis loop of ferromagnetic materials (Barkhausen noise), and simple enough to allow exact analytical results $^{22,23,41-43}$. It predicts power law distributions for avalanche size and duration, as well as parabolic average avalanche shape in the scaling regime, and has inspired the extant models for intermittent granular flow ${ }^{4,44}$. We will discuss the connections between this model and what we observe in our study in the conclusive section.

Average time profile of the slip velocity. To investigate the properties of the average time profile of the velocity, and to test the hypothesis of scaling invariance, we have divided all the avalanches observed in the experiments into classes according to their duration (see Mehods and Supplementry information). Figure 3 (main panel) shows the size of each slip as function of its duration, for all the slips considered in the statistics. Different 

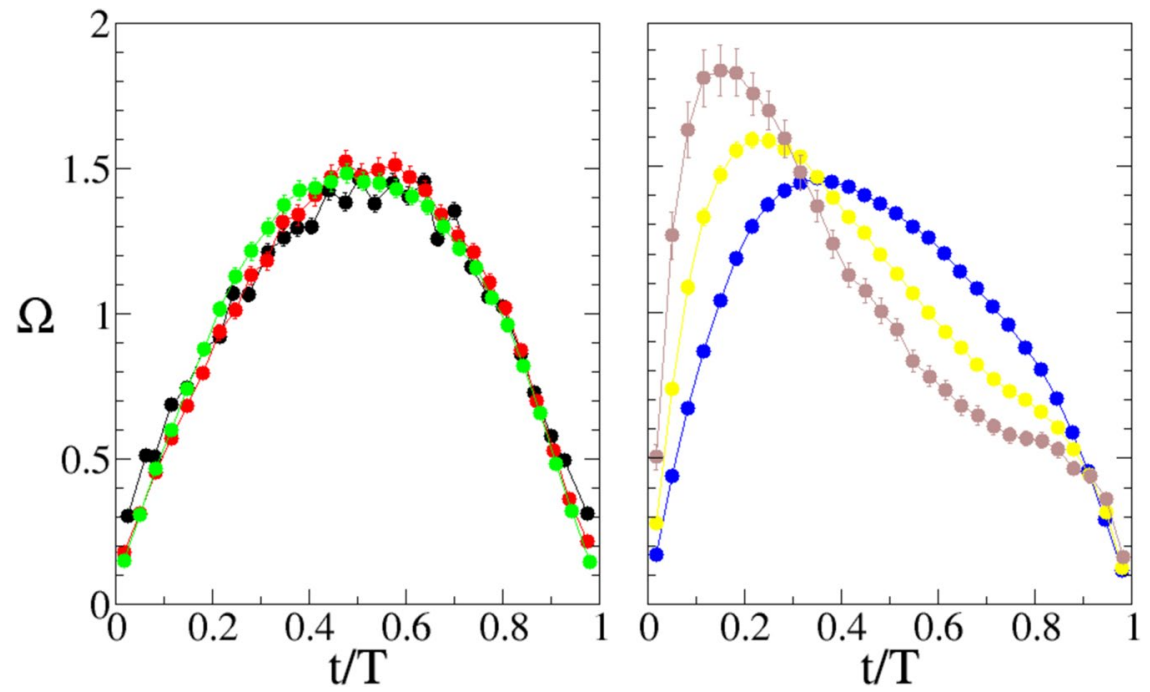

Figure 4. Time profiles of the normalized average velocity profile during a slip for "short" and "long" avalanches, as a function of the internal time $t / T$ (Eqs 1 and 4). Average profiles are computed over avalanches belonging to a same class of duration, shown in the scatter plot of Fig. 3 with the same symbol coding (see SI for the precise definition). The left panel shows the average velocity profile for "short" avalanches (first three classes), and their very good scaling, while the right panel shows the profile for "long avalanches" (last three classes), where the scaling is broken.

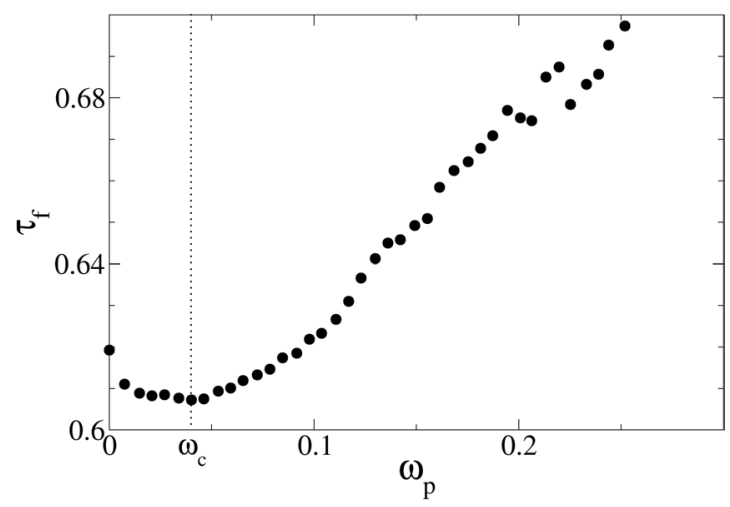

Figure 5. Experimental measure of the average friction torque at a given instantaneous plate velocity $\omega_{p}$ (defined in Eq. 5). The curve displays weakening behavior before reaching a minimum value at a characteristic velocity $\omega_{c} \approx 0.04 \mathrm{rad} / \mathrm{s}$, after which friction increases for increasing plate velocities.

colors and symbols correspond to the different classes of duration. For each class we have computed the rescaled average velocity time profile, or shape $\Omega$ of Eq. 1 (see Methods and Supplementry information). The resulting profiles are shown in Fig. 4 (light, gray points in Fig. 3, corresponding to very short slips at the limit of the system resolution, have not been considered). In the scaling scenario $\Omega$ is a function of $t / T$ only, and it is seen that all classes exhibit comparable values of the rescaled maximum velocity, implying that longer avalanches are also faster. However, average shapes unveil that there are two kinds of avalanches. Some of them, say short, have the shape described by a unique function $\Omega(t / T)$, visible in Fig. 4 (left panel). That is, their rescaled velocity profile and duration are related by the well defined scaling law Eq. 1 . On the contrary, the average velocity shapes of long avalanches (right panel) change with the duration and cannot be reduced to a universal form by a homogeneous rescaling of the variables. Moreover, they do not display the almost symmetric shape characterizing small avalanches.

As anticipated, Barés and coworkers ${ }^{38}$ have recently measured the average shape of stress drop rate avalanches in a bidimensional granular system driven at constant shear rate. Similarly to the present findings, they have observed that longer slips develop left asymmetries. They have hypothesized a possible role of the static friction between particles and supporting glass, and of nonlinear elasticity, given by the relatively soft nature of the grain material employed in their experiments. We can however exclude these factors in our experiments, where the interface grain-wall is small with respect to the bulk and the beads are made of a rather stiff material like glass. The leftwards asymmetry observed in experiments represents a genuine and very interesting phenomenon, which 
cannot be due to the simple inertia of the moving plate (which should produce opposite asymmetry ${ }^{20,21}$ ) and originates from non trivial dynamical effects.

Breaking of scaling. More insight into the mechanisms leading to the scaling breakdown can be gained by further analyzing the scatter plot relating $S$ and $T$ in Fig. 3. The first information coming from this plot is that there exists a definite statistical scaling between slip size and duration. The white squared symbols in the main plot represent the average slip size and duration of each class (statistical errors are negligible on these averages). It is seen that for the four lower classes they follow an algebraic relation: $\langle S\rangle_{j} \simeq\langle T\rangle_{j}^{\alpha}$ (red continuous line). The value of the exponent turns out to be $\alpha \simeq 2.2$ (which is close to, but clearly different from the value of $\alpha=2$ expected from extant models, as for instance the ABBM model mentioned above ${ }^{41}$ ). The second information supplied by the scatter plot of Fig. 3 is that this behavior changes for long slips, where a linear dependence, $\langle S\rangle \propto\langle T\rangle$ looks more appropriate (yellow dashed line). Notice that the crossover between the two behaviors takes place around the fourth class, exactly where the scaling of the average pulse shape breaks down in Fig. 4. This fact is put into better evidence in the inset of Fig. 3, where we have plotted the average velocity of the whole slip $\langle S\rangle_{j} /\langle T\rangle_{j}$, as function of $\langle T\rangle_{j}$. Here a weakly superlinear relation for short slips is followed by a plateau at long slips. This allows to identify a characteristic velocity $\omega_{c}$, as the ratio between the average slip size and the average duration of the fourth class. It results $\omega_{c}=\left\langle S_{c}\right\rangle /\left\langle T_{c}\right\rangle \approx 0.063 / 1.57 \approx 0.04 \mathrm{rad} / \mathrm{s}$. The slowdown of large avalanches suggests an increase of friction in long slips $\left(T>T_{c}\right)$, when the plate reaches velocities $\omega_{p}>\omega_{c}$. In the next section it will be seen that indeed this increase appears as a dynamical effect.

Stochastic friction. Friction in granular systems is usually measured under controlled shear strain or stress. The classical Mohr-Coulomb criterion predicts constant friction at low shear, and increasing values when the system enters the Bagnold's regime ${ }^{45,46}$, a behavior well observed experimentally at constant shear (see e.g. ${ }^{47}$ ). However, it is doubtful whether this description applies to the stick-slip dynamics exemplified in Fig. 1. As a matter of fact, in the stick-slip regime, friction is a random quantity. Fluctuations in the frictional response of the granular medium result from the stress propagation along the evolving network of grain contacts, and are at the very origin of the motion stochasticity. Some statistical features of friction in this state have been investigated ${ }^{3-5}$, but despite this quantity plays a crucial role for the system dynamics, it seems that it has never been systematically measured during stick slip to date.

A random friction force, as a stochastic quantity, can be described by statistical estimators like averages, moments, correlators, etc. One can consider for example the time average of the friction over the full dynamics, but this does not look really meaningful. Another possibility ${ }^{4,48}$ is to consider the average friction as function of the instantaneous plate velocity (see Methods). We plot such conditioned average friction, $\tau_{f}$, during the stick slip regime, in Fig. 5. An interesting weakening of friction at small velocities can be observed, followed by a thickening which recovers the Bagnold behavior at high velocities, a behavior also known as Stribeck curve. This velocity weakening arises as a pure dynamical effect since at constant shear the average friction is constant at low and intermediate speeds ${ }^{47}$.

Figure 5 allows to identify a velocity corresponding to the position of the minimum of the average friction $\tau_{f}$. It is always attained near the $\omega \approx 0.04 \mathrm{rad} / \mathrm{s}$, and our experiments clearly indicate that the position of this minimum does not depend on the drive velocity (see figures in the SI). This value is very close to the value $\omega_{c}$ marking the crossover in the scaling of $S$ vs $T$ in Fig. 3, and the breakdown of shape self-similarity in the average avalanche (Fig. 4), reinforcing the idea that both originate from the different friction experienced by the plate during longer, faster avalanches.

In order to better understand whether and how friction dynamical behavior can influence the average velocity shape, we have analyzed also the average shape of friction along the slip. In analogy to what has been done for computing the velocity shapes, one can define $\langle\tau(t)\rangle_{T}$ as the average frictional torque for slips of the same duration $T$. In practice, we have computed the average value of the friction torque during slips of similar duration $T$, according to the same classes of duration adopted for velocities (Fig. 3 and Supplementry information). The results are presented in Fig. 6 and show that to the break of the velocity shape scaling, it corresponds a change in the properties of the average friction shape. In scaling avalanches (left graph of Fig. 6) the average friction is largely independent from the slip duration and almost constant along the whole slip. On the contrary, the averages corresponding to longer slips (right graph of Fig. 6) display different shapes that, as for velocity (Fig. 4), strongly depend on $T$ and cannot be collapsed. Moreover, in comparison with short slips, longer slips display higher friction at the beginning of the slip and lower at the end.

Let us stress here the difference between the two averages considered above (Figs 5 and 6). The average $\langle\tau\rangle_{T}$ shown in Fig. 6, is performed over slips of similar duration, at the same internal avalanche time $t$. Instead, the (conditional) average $\tau_{f}$, defined in Eq. 5 and shown in Fig. 5, mixes events of any duration and depends on the instantaneous plate velocity $\omega_{p}$. The two quantities give different aspects of the same (stochastic) physical phenomenon. Nevertheless, the combination of the two analysis indicates that the quite complex friction weakening behavior of $\tau_{f}$ is mainly due to long slips, which show a non constant average friction $\langle\tau\rangle_{T}$ in time (Fig. 6, right panel) in contrast with small slips, where the average stays mainly constant. This point becomes still more evident by considering the curves resulting from combining the analysis of friction and velocity shapes, plotting the average friction as a function of the average velocity as shown in Fig. 7. It is seen that while in small slips (left panel), friction has a low velocity dependence (as could be expected from Fig. 6), in long ones it displays hysteresis, splitting into a two-valued function (right panel) with different dependency on velocity in the accelerating and decelerating phase, similarly to what observed in periodic stick-slip ${ }^{49,50}$ (where all slips have identical extension, duration, and velocity profile). 

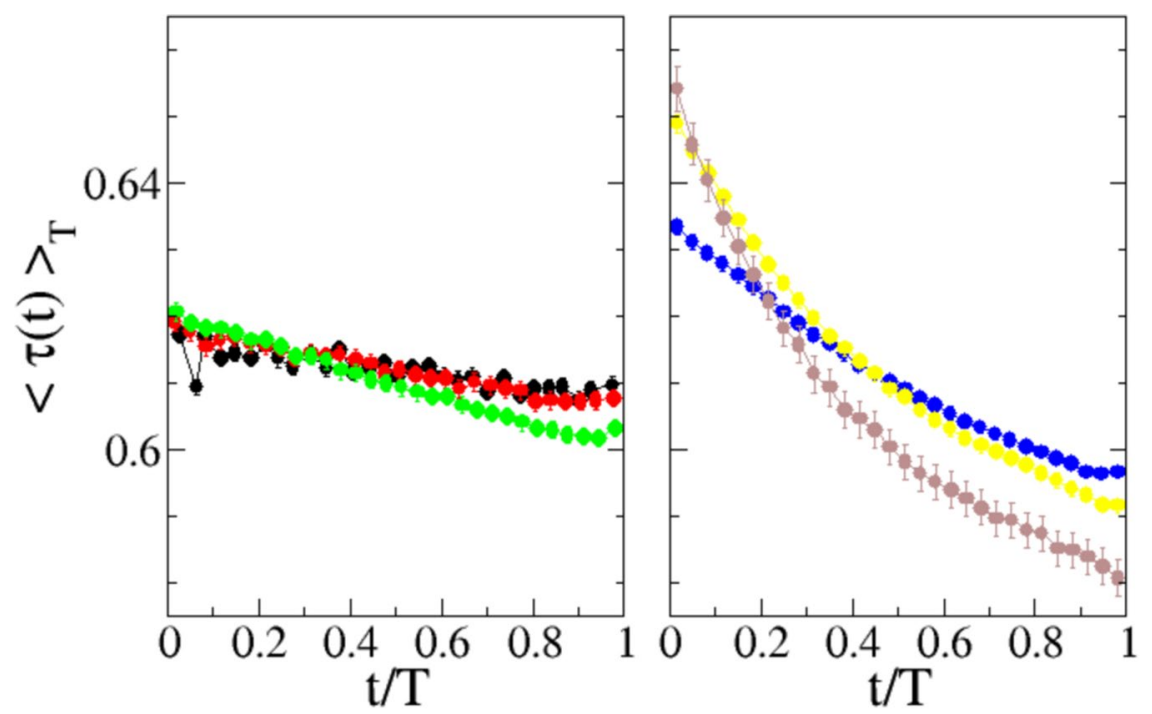

Figure 6. Experimental measure of the average friction torque along slips of different duration, as function of the internal time. The averages are performed grouping avalanches into classes according to their duration (see SI for the definition). Each curve refers to a different class shown with same colors and symbols used in Figs 3 and 4. Left panel shows the average friction for "short" avalanches (first three classes), while right panel shows the behavior for "long" avalanches (last three classes).
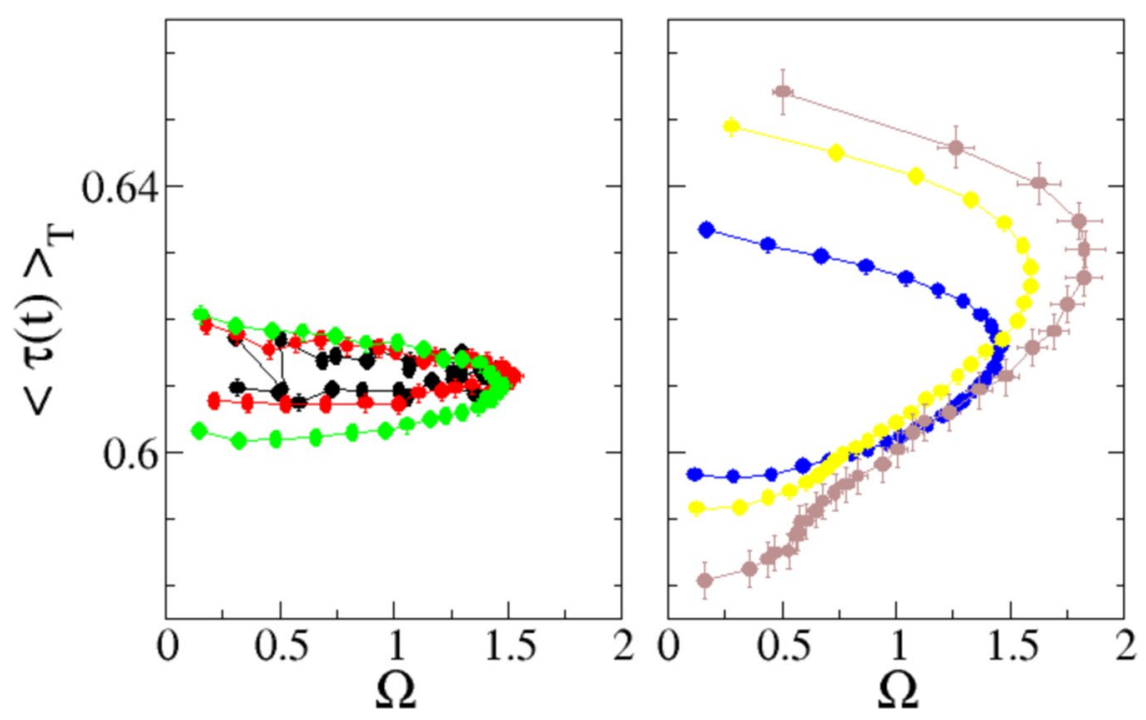

Figure 7. Average friction torque along slips of different duration as a function of the normalized average slip velocity. The plot is a parametric combination of Figs 4 and 6. The averages are performed over avalanches of similar duration, according to six classes of increasing duration (see SI for the definition). Each symbol refers to a different class withe the same coding used in Figs 3, 4 and 6. Left panel shows the behavior for "short" avalanches (first three classes), while right panel shows the behavior for "long" avalanches (last three classes).

\section{Discussion}

Our experiments show good scaling of the average velocity time profile in short avalanches, with an almost symmetric shape. In longer avalanches however, profiles take a clear leftward asymmetry and scaling, Eq. 1, is broken. A similar phenomenology has been observed also in a $2 d$ system $^{51}$, and in earthquakes ${ }^{36,52}$, where it is not yet clear the way different mechanisms can contribute to the shear weakening observed in coseismic fault shearing ${ }^{53}$. Our analyses show that the breakdown of scaling takes place along with a change in the friction behavior, pointing out a strict relation between the two phenomena. These results are summarized in Fig. 8, where all the slips of the present analysis are reported. For each slip the plot shows the instantaneous velocity ( $z$ surface) and the corresponding instantaneous friction (bottom map), as function of the internal time $t / T$ and of the avalanche duration $T$ (data have undergone a standard interpolation in order to get a smoother surface). For both quantities, velocity and friction, colors represent the relative magnitude, from black (minimum values) to green (maximum values). The $z$ values of instantaneous velocity are normalized to the average velocity during the avalanche. It is seen that 


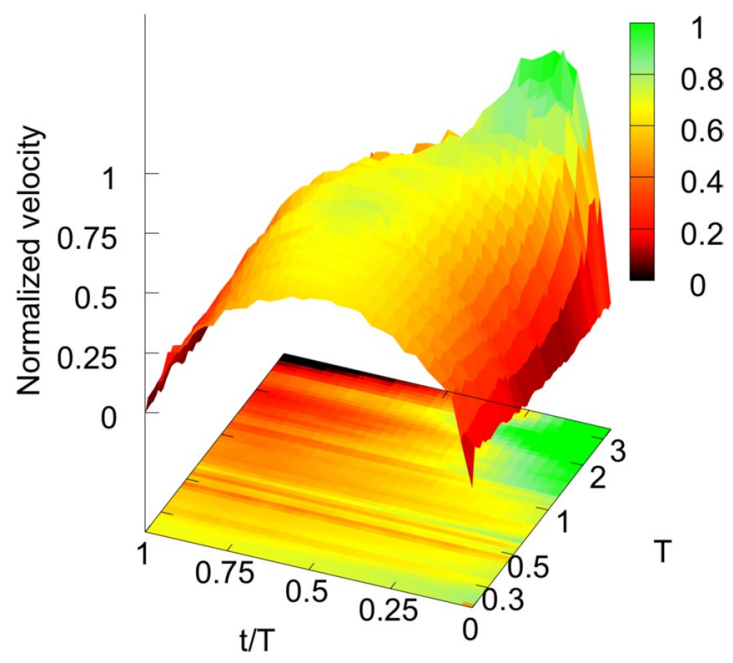

Figure 8. The figure shows the instantaneous velocity (upper surface) and the instantaneous friction (bottom plane) of each single slip observed in the experiment, as functions of the avalanche duration $T$ and of its internal time $t / T$. The instantaneous velocity of each slip has been normalized by the average velocity during the slip, and then by a factor common to all slips such that the resulting velocities span between 0 and 1 (shown by the colors on the surface). Note how the "short" avalanches display a simmetric, scaling profile, while for "long" avalanches, the scaling is broken and the profiles get more and more asymmetric. The instantaneous friction, globally rescaled in order to get values spanning between 0 (minimum friction) to 1 (maximal friction), is represented with the color map in the botton plane. Note that the instantaneous friction for "short" avalanches keeps quite constant, while for "long" avalanches decreases markedly during the avalanches.

a relatively constant and moderate value of friction, colored from yellow to orange, corresponds to the symmetric shape of short avalanches. On the contrary, longer asymmetric avalanches are related to decreasing values (in time) of the friction. In particular, the lowest friction values (black) are typically recorded in the final part of long $\operatorname{slips}(t / T \approx 1)$, while the initial part $(t / T \approx 0)$ displays the highest values (green).

The figure illustrates in a synthetic way the complexity of the frictional response of the granular bed, and highlights the necessity of considering suitable friction laws to model such phenomenology in effective way (spring-block models with imposed Coulomb friction generate symmetric slips ${ }^{54,55}$ ). On the other hand ${ }^{19}$, there is a strict relationship between avalanche shape symmetry and time reversibility, and this is in turn related to system non Markovianity, or memory. Friction laws working well for stationary flows ${ }^{56}$ cannot account for phenomena observed in non stationary situations, like for instance hysteresis. In the past, phenomenological friction laws accounting for the elapsed time and/or space along the slip have been derived from solid-on-solid experiment $^{57-59}$. Although showing a limited validity for interstitial granular matter ${ }^{60,61}$, these rate-and-state laws and their simplified forms are often adopted for studying and modeling co-seismic fault shearing, ${ }^{55,62-64}$. Attempts of incorporating them to model stick slip experiments seems very rare ${ }^{48}$ and would be desirable.

An effective modeling approach to the stick-slip granular dynamics cannot as well exclude a stochastic description of friction, which generates slip unpredictability and variability, with the consequent change in the slip shapes. To our knowledge, the only few attempts in this direction ${ }^{4,44,48}$ are inspired to the aforementioned $A B B M$ model $^{41}$, which represents the mean field approximation for the motion of a driven elastic interface in a random environment ${ }^{40,65}$. From the dynamical point of view the model describes a spring-slider system in the overdamped approximation. (i.e. with negligible inertia) and subject to a friction which includes both a viscous force and a random pinning force. At small driving rate, the ABBM model produces intermittent, self-similar dynamics for the block motion. Similarly to our observations, avalanche statistics show a scaling regime for short slips, whose average velocity has parabolic shapes. However, an exponent $\alpha=2$ relates $\langle S\rangle$ to $\langle T\rangle$, which is different from what observed in our experiments. Moreover, for longer slips, ABBM predicts flatter but still symmetrical average velocity shapes, since no inertial effects are present. A variant of the ABBM model including inertia has shown to well reproduce the granular slip statistics ${ }^{4}$ in terms of extension, duration and velocities. Velocity distributions for an inertial ABBM model have also been studied by Le Doussal et al. ${ }^{6}$. Stimulated by the present results, investigations of the velocity shape generate by this model are in progress.

Finally, non trivial inertial effects should be considered. For instance, in some experiments ${ }^{50}$ an increased inertia of the slider moving on a granular bed was found, due to the grains dragged by the slider itself. In our previous experiments ${ }^{4}$ an effect of augmented inertia has been observed (in the scaling of the peak in the duration distribution). However, since during the irregular motion of the system the quantity of grains dragged by the disk could change, one should consider the inertia as a dynamical quantity, rather than a constant. The existence of such "effective" dynamical inertia of the system is another form of memory introduced by the underlying granular dynamics. An instance of such a mechanism, leading to a leftwards asymmetry in the avalanche shape, has been observed in some magnetic materials ${ }^{67}$ where it results from an effective negative mass of the domain walls. Grain inertia can influence the avalanche statistics even at the microscopic level. In sandpile models, largely studied in 
the context of Self Organized Criticality (SOC), the tendency of real sand grains to keep moving once they start facilitates the emergence of huge avalanches. This has been proven also experimentally, where small non inertial avalanches have been shown to have well distinct properties from large inertial ones ${ }^{68,69}$. Recent theoretical developments propose, in the presence of such facilitation effects, a scenario called Self-Organised Bistability $(\mathrm{SOB})^{70,71}$, where again a breaking of scaling is associated to the appearance of large avalanches ("kings").

In conclusion, our detailed scaling analyses show that symmetric and asymmetric average avalanche shapes are both present, but refer to different sizes, "short" vs "long", and corresponding frictional regimes, explaining contradictory recent observations ${ }^{37,38}$. In addition, the study demonstrates that granular friction can exhibit weakening also in the vicinity of the jamming transition. Randomness and memory are general features that cannot be overlooked in the formulation of effective models of physical phenomena involving granular flow, as landslides and earthquakes, and of crackling noise in general.

\section{Methods}

The experimental set up is described in detail in the SI and is similar to that employed in previous experiments $^{3-5,48,72}$. It consists of an assembly of glass spheres laying in an annular channel and sheared by a horizontally rotating top plate driven by a motor. The instantaneous angular position of the plate and of the motor, respectively $\theta_{p}$ and $\theta_{d}$ are acquired by means of two optical encoders. The plate is coupled to the motor through a soft torsion spring of elastic constant $k$. The instantaneous frictional torque, $\tau$, exerted by the granular medium can be derived from the equation of motion for the plate:

$$
\tau=-k\left(\theta_{d}-\theta_{p}\right)-I \ddot{\theta}_{p},
$$

where $I$ is the inertia of the plate-axis system. The motor angular speed $\omega_{d}$ is kept constant, so that $\theta_{d}(t)=\omega_{d} t$.

To compute the average slip one can integrate Eq. (1) with respect to $t$ obtaining:

$$
\langle S\rangle_{T}=\operatorname{Tg}(T)
$$

(where without loss of generality we have assumed $\int_{0}^{1} \Omega(x) d x=1$ ). The function $\Omega$ represents the average invariant pulse shape, which is expected not to depend on the slip duration and can be computed via the above equations as

$$
\Omega(t / T)=T \frac{\left\langle\omega_{p}(t)\right\rangle_{T}}{\langle S\rangle_{T}} .
$$

After classifying the slips according to their duration (as described in the subsection "Average shape of slip velocity" and in the SI), we have computed for each class, $j$, the average slip size $\langle S\rangle_{j}$ and duration $\langle T\rangle_{j}$, and the average velocity $\left\langle\omega_{p}(t)\right\rangle_{j}$ measured as function of the internal time $t$. In order to obtain $\Omega(t / T)$, this average velocity has then been normalized to the ratio $\langle S\rangle_{j} /\langle T\rangle_{j}$, according to Eq. 4.

The average friction as function of the instantaneous plate velocity $\omega_{p}(t)$, discussed in the subsection "Stochastic friction" and shown in Fig. 5, has been computed as the average friction conditioned to the vaule of $\omega_{p}(t)$ :

$$
\tau_{f}(\omega)=\lim _{T \rightarrow \infty} \frac{\int_{0}^{T} \tau(t) \delta\left(\omega-\omega_{p}(t)\right) d t}{\int_{0}^{T} \delta\left(\omega(t)-\omega_{p}\right) d t} .
$$

Received: 8 April 2019; Accepted: 11 October 2019;

Published online: 18 November 2019

\section{References}

1. Jaeger, H. M., Shinbrot, T. \& Umbanhowar, P. B. Does the granular matter? Proceedings of the National Academy of Sciences 97, $12959-12960$ (2000).

2. Dalton, F. \& Corcoran, D. Self-organized criticality in a sheared granular stick-slip system. Physical Review E 63, 061312, https://doi. org/10.1103/PhysRevE.63.061312 (2001).

3. Dalton, F. et al. Shear stress fluctuations in the granular liquid and solid phases. Phys. Rev. Lett. 95, 138001 (2005).

4. Baldassarri, A. et al. Brownian forces in sheared granular matter. Physical Review Letters 96, 118002 (2006).

5. Petri, A. et al. Stochastic dynamics of a sheared granular medium. The European Physical Journal B - Condensed Matter and Complex Systems 64, 531-535 (2008).

6. Pica Ciamarra, M., Lippiello, E., Godano, C. \& de Arcangelis, L. Unjamming dynamics: the micromechanics of a seismic fault model. Physical review letters 104, 238001 (2010).

7. Geller, D. A., Ecke, R. E., Dahmen, K. A. \& Backhaus, S. Stick-slip behavior in a continuum-granular experiment. Phys. Rev. E 92, 060201, https://doi.org/10.1103/PhysRevE.92.060201 (2015).

8. Annunziata, M. A., Baldassarri, A., Dalton, F., Petri, A. \& Pontuale, G. Increasing 'ease of sliding' also increases friction: when is a lubricant effective? Journal of Physics: Condensed Matter 28, 134001 http://stacks.iop.org/0953-8984/28/i=13/a=134001 (2016).

9. Zadeh, A. A., Barés, J., Socolar, J. E. S. \& Behringer, R. P. Seismicity in sheared granular matter. preprint arXiv:1810.12243v1[condmat.soft (2018).

10. Sethna, J. P., Dahmen, K. A. \& Myers, C. R. Crackling noise. Nature 410, 242-250 (2001).

11. Main, I. Statistical physics, seismogenesis, and seismic hazard. Reviews of Geophysics 433-462 (1996)

12. Petri, A., Paparo, G., Vespignani, A., Alippi, A. \& Costantini, M. Experimental evidence for critical dynamics in microfracturing processes. Phys. Rev. Lett. 73, 3423-3426 (1994).

13. Cannelli, G., Cantelli, R. \& Cordero, F. Self-organized criticality of the fracture processes associated with hydrogen precipitation in niobium by acoustic emission. Phys. Rev. Lett. 70, 3923-3926 (1993). 
14. Dimiduk, D. M., Woodward, C., LeSar, R. \& Uchic, M. D. Scale-free intermittent flow in crystal plasticity. Science 312, 1188-1190 (2006).

15. Bak, P., Tang, C. \& Wiesenfeld, K. Self-organized criticality. Phys. Rev. A 38, 364-374 (1988).

16. Dauchot, O., Marty, G. \& Biroli, G. Dynamical heterogeneity close to the jamming transition in a sheared granular material. Phys. Rev. Lett. 95, 265701 (2005).

17. Bohn, F. et al. Playing with universality classes of barkhausen avalanches. Scientific reports 8, 11294 (2019).

18. Kuntz, M. C. \& Sethna, J. P. Noise in disordered systems: The power spectrum and dynamic exponents in avalanche models. Physical Review B - Condensed Matter and Materials Physics 62, 11699-11708 9911207 (2000).

19. Laurson, L. et al. Evolution of the average avalanche shape with the universality class. Nat. Commun. 4 (2013).

20. Baldassarri, A., Colaiori, F. \& Castellano, C. Average Shape of a Fluctuation: Universality in Excursions of Stochastic Processes. Physical Review Letters 90, 60601 http://link.aps.org/abstract/PRL/v90/e060601 (2003).

21. Colaiori, F., Baldassarri, A. \& Castellano, C. Average trajectory of returning walks. Physical Review E (Statistical, Nonlinear, and Soft Matter Physics) 69, 41105 http://link.aps.org/abstract/PRE/v69/e041105 (2004).

22. Colaiori, F. Exactly solvable model of avalanches dynamics for barkhausen crackling noise. Advances in Physics 57, 287-359 (2008).

23. Papanikolaou, S. et al. Universality beyond power laws and the average avalanche shape. Nature Physics 7, 316-320, https://doi. org/10.1038/nphys1884 (2011).

24. Chrzan, D. C. \& Mills, M. J. Criticality in the plastic deformation of 112 intermetallic compounds. Physical Review B 50, 30 (1994).

25. Sparks, G., Sickle, J., Dahmen, K. \& Maas, R. Shapes and velocity relaxation of dislocation avalanches in fcc and bcc crystals. arXiv:1705.06636 (2017).

26. Antonaglia, J. et al. Bulk metallic glasses deform via slip avalanches. Phys. Rev. Lett. 112, 155501 (2014).

27. Liu, C., Ferrero, E. E., Puosi, F., Barrat, J.-L. \& Martens, K. Driving rate dependence of avalanche statistics and shapes at the yielding transition. Phys. Rev. Lett. 116, 065501, https://doi.org/10.1103/PhysRevLett.116.065501 (2016).

28. Lagogianni, A. E., Liu, C., Martens, K. \& Samwer, K. Plastic avalanches in the so-called elastic regime of metallic glasses. The European Physical Journal B 91, 104, https://doi.org/10.1140/epjb/e2018-90051-7 (2018).

29. Roberts, J. A., Iyer, K. K., Finnigan, S., Vanhatalo, S. \& Breakspear, M. Scale-Free Bursting in Human Cortex following Hypoxia at Birth. The Journal of Neuroscience 34, 6557 LP - 6572 http://www.jneurosci.org/content/34/19/6557.abstract (2014).

30. Wikstro, S. et al. Cortical burst dynamics predict clinical outcome early in extremely preterm infants. Brain 138, 2206-2218 (2015).

31. Wang, B., Kuo, J. \& Granick, S. Bursts of active transport in living cells. Phys. Rev. Lett. 111, 208102, https://doi.org/10.1103/ PhysRevLett.111.208102 (2013).

32. Gallotti, R. \& Chialvo, D. R. How ants move: individual and collective scaling properties. Journal of The Royal Society Interface 15 http://rsif.royalsocietypublishing.org/content/15/143/20180223.abstract (2018).

33. Chialvo, D. et al. How we move is universal: Scaling in the average shape of human activity. Papers in Physics 7 http://www. papersinphysics.org/papersinphysics/article/view/275 (2015).

34. Sheikh, M. A., Weaver, R. L. \& Dahmen, K. A. Avalanche statistics identify intrinsic stellar processes near criticality in kic 8462852. Phys. Rev. Lett. 117, 261101, https://doi.org/10.1103/PhysRevLett.117.261101 (2016).

35. Consolini, G., De Michelis, P. \& Tozzi, R. On the Earth's magnetospheric dynamics: Nonequilibrium evolution and the fluctuation theorem. Journal of Geophysical Research: Space Physics 113, 1-11 (2008).

36. Mehta, A. P., Dahmen, K. A. \& Ben-Zion, Y. Universal mean moment rate profiles of earthquake ruptures. Phys. Rev. E 73, 056104, https://doi.org/10.1103/PhysRevE.73.056104 (2006).

37. Denisov, D. V. et al. Universal slip dynamics in metallic glasses and granular matter - linking frictional weakening with inertial effects. Scientific Reports 7, 43376 http://www.nature.com/articles/srep43376 (2017).

38. Barés, J. et al. Local and global avalanches in a 2d sheared granular medium. Phys. Rev. E 96, 052902 http://arxiv.org/abs/1709.01012. 1709.01012 (2017).

39. Fisher, M. E. The theory of equilibrium critical phenomena. Reports on Progress in Physics 30, 615 http://stacks.iop.org/0034$4885 / 30 / \mathrm{i}=2 / \mathrm{a}=306(1967)$

40. Le Doussal, P. \& Wiese, K. J. Distribution of velocities in an avalanche. Europhys. Lett. 97, 460041104.2629 (2012).

41. Alessandro, B., Beatrice, C., Bertotti, G. \& Montorsi, A. Domain-wall dynamics and barkhausen effect in metallic ferromagnetic materials. i. theory. Journal of Applied Physics 68, 2901, https://doi.org/10.1063/1.346423/html (1990).

42. Feller, W. Two singular diffusion problems. Annals of mathematics 54, 173-182 (1951).

43. Dobrinevski, A., Le Doussal, P. \& Wiese, K. J. Nonstationary dynamics of the Alessandro-Beatrice-Bertotti-Montorsi model. Physical Review E - Statistical, Nonlinear, and Soft Matter Physics 85, 1-16 1112.6307 (2012).

44. Dahmen, K. A., Ben-Zion, Y. \& Uhl, J. A simple analytic theory for the statistics of avalanches in sheared granular materials. Nature Physics 1957 (2011).

45. Bagnold, R. A. Experiments on a gravity-free dispersion of large solid spheres in a newtonian fluid under shear. Proc. Roy. Soc. A 225, 49-63 (1954)

46. Bagnold, R. A. The shearing and dilatation of dry sand and the 'singing' mechanism. Proc. Roy. Soc. A 295, 219-232 (1965).

47. Savage, S. B. \& Sayed, M. Stresses developed by dry cohesionless granular materials shared in an annular shear cell. J. Fluid Mech. 142, 391-430 (1984).

48. Leoni, F. et al. Friction memory in the stick-slip of a sheared granular bed. Journal of Non-Crystalline Solids 357, 749-753 (2010).

49. Nasuno, S., Kudrolli, A. \& Gollub, J. P. Friction in granular layers: Hysteresis and precursors. Phys. Rev. Lett. 79, 949-952 (1997).

50. Nasuno, S., Kudrolli, A., Bak, A. \& Gollub, J. P. Time-resolved studies of stick-slip friction in sheared granular layers. Phys. Rev. E 58, 2161-2171 (1998).

51. Barés, J., Hattali, M. L., Dalmas, D. \& Bonamy, D. Fluctuations of global energy release and crackling in nominally brittle heterogeneous fracture. Phys. Rev. Lett. 113, 264301, https://doi.org/10.1103/PhysRevLett.113.264301 (2014).

52. Houston, H., Benz, H. M. \& Vidale, J. E. Time functions of deep earthquakes from broadband and short-period stacks. Journal of Geophysical Research 103, 29895 (1998).

53. Di Toro, G. et al. Earthquakes and friction laws. Nature 471, 7339 (2011).

54. Aharonov, E. \& Spark, D. Stick-slip motion in simulated granular layers. Journal of Geophysical Research: Solid Earth 109 (2004)

55. Bizzarri, A. \& Petri, A. Single slip dynamics. Physics of the Earth and Planetary Interiors 261, 36-45 http://www.sciencedirect.com/ science/article/pii/S0031920116301236 (2016)

56. GDRMiDi2004. Dense flows of dry granular material: écoulements de milieux granulaires secs. Europhysics Journal 14, 341 (2004).

57. Ruina, A. Slip instability and state variable friction laws. Journal of Geophysical Research: Solid Earth 88, 10359-10370 (1983).

58. Dieterich, J. A constitutive law for rate of earthquake production and its application to earthquake clustering. Journal of Geophysical Research: Solid Earth 99, 2601-2618 (1994).

59. Baumberger, T. \& Caroli, C. Solid friction from stick-slip down to pinning and aging. Advances in Physics 55, 279-348 (2006).

60. Mair, K. \& Marone, C. Friction of simulated fault gouge for a wide range of velocities and normal stresses. Journal Geophysical Research 104, 899-914 (1999).

61. Leeman, J., Marone, C. \& Saffer, D. Frictional mechanics of slow earthquakes. Journal of GeophysicalResearch: Solid Earth 123 (2018).

62. Scholz, C. H. Earthquakes and friction laws. Nature 391, 37 (1998).

63. Bizzarri, A. \& Cocco, M. Slip-weakening behavior during the propagation of dynamic ruptures obeying rate- and state-dependent friction laws. Journal of Geophysical Research: Solid Earth 108, 2373 (2003). 
64. Kawamura, H., Hatano, T., Kato, N., Biswas, S. \& Chakrabarti, B. K. Statistical physics of fracture, friction, and earthquakes. Rev. Mod. Phys. 84, 839-884, https://doi.org/10.1103/RevModPhys.84.839 (2012).

65. Zapperi, S., Cizeau, P., Durin, G. \& Stanley, H. E. Dynamics of a ferromagnetic domain wall: Avalanches, depinning transition, and the Barkhausen effect. Physical Review B 58, 6353-6366, https://doi.org/10.1103/PhysRevB.58.6353 9803253 (1998).

66. Le Doussal, P., Petković, A. \& Wiese, K. J. Distribution of velocities and acceleration for a particle in Brownian correlated disorder: Inertial case. Physical Review E 85, 061116, https://doi.org/10.1103/PhysRevE.85.061116. 1203.5620 (2012).

67. Zapperi, S., Castellano, C., Colaiori, F. \& Durin, G. Signature of effective mass in crackling-noise asymmetry. Nature Physics 1, 46-49 (2005).

68. Nerone, N. \& Gabbanelli, S. Surface fluctuations and the inertia effect in sandpiles. Granular Matter 3, 117-120 (2000).

69. Nerone, N., Aguirre, M., Calvo, A., Bideau, D. \& Ippolito, I. Instabilities in slowly driven granular packing. Physical Review E 67, $011302(2003)$

70. Di Santo, S., Burioni, R., Vezzani, A. \& Muñoz, M. A. Self-Organized Bistability Associated with First-Order Phase Transitions. Physical Review Letters 116, 1-5 1605.05161 (2016).

71. Di Santo, S., Villegas, P., Burioni, R. \& Muñoz, M. Landau-ginzburg theory of cortex dynamics: Scale-free avalanches emerge at the edge of synchronization. Proceedings of the National Academy of Sciences 115, E1356 (2018).

72. Pica Ciamarra, M. et al. The role of interstitial impurities in the frictional instability of seismic fault models. Tribology Letters 48, 89-94 (2012).

\section{Acknowledgements}

This work has been supported by the grant FIRB RBFR081IUK_003. The authors also acknowledge the financial support of Regione Lazio through the Grant "Progetti Gruppi di Ricerca” N. 85-2017-15257.

\section{Author contributions}

A.B. and A.P. designed research. A.G. and G.P. modified and improved the experimental set-up. A.G., A.P. and G.P. performed the experiments. M.A.A. analyzed data, A.B. and A.P. analyzed the data and wrote the paper.

\section{Competing interests}

The authors declare no competing interests.

\section{Additional information}

Supplementary information is available for this paper at https://doi.org/10.1038/s41598-019-53178-2.

Correspondence and requests for materials should be addressed to A.P.

Reprints and permissions information is available at www.nature.com/reprints.

Publisher's note Springer Nature remains neutral with regard to jurisdictional claims in published maps and institutional affiliations.

Open Access This article is licensed under a Creative Commons Attribution 4.0 International License, which permits use, sharing, adaptation, distribution and reproduction in any medium or format, as long as you give appropriate credit to the original author(s) and the source, provide a link to the Creative Commons license, and indicate if changes were made. The images or other third party material in this article are included in the article's Creative Commons license, unless indicated otherwise in a credit line to the material. If material is not included in the article's Creative Commons license and your intended use is not permitted by statutory regulation or exceeds the permitted use, you will need to obtain permission directly from the copyright holder. To view a copy of this license, visit http://creativecommons.org/licenses/by/4.0/.

(C) The Author(s) 2019 